If an algebraic surface $F$ of order $\nu \geqq 4$ has a double line (either nodal or cuspidal), the anomaly $A$ of the plane sections $C$ of a tangent cone to $F$ is $A=(1 / 6) \nu(\nu-4)(\nu-5)$. As is evident from this expression for $A$, plane curve systems $C$ associated with a surface having a double line are anomalous only for $\nu \geqq 6$.

When $\nu=3$, the above formula yields $A=1$. However, a cubic surface with a nodal line is ruled, and a cubic surface with a cuspidal line is a cone. The treatment given in the two preceding sections and the resulting expression for $A$ do not apply to such surfaces.

\title{
Wells College
}

\section{ON THE STABILITY OF THE LAMINAR FLOW OF A VISCOUS FLUID ${ }^{1}$}

\author{
RUDOLPH E. LANGER
}

The problem of the effect of two-dimensional first-order disturbances upon the laminar flow of an incompressible viscous fluid is known to be fundamental to the analysis of the phenomenon of turbulence. This discussion is concerned with such a problem in the case of a flow which takes place parallel to and between parallel plane boundaries. If the direction normal to these boundaries is designed by $y$, and that of the flow by $x$, the unit of length and the origin of coordinates may be chosen so that the boundary planes are given by the equations $y=1$ and $y=-1$. It is to be assumed then that the velocity of the undisturbed flow depends only on $y$, and is given by a function $U(y)$, which is suitably differentiable and nonnegative, which is nonincreasing as to $|y|$, and which vanishes at the boundaries. If the maximum velocity is chosen as the unit, as will be assumed, it follows that

$$
U(0)=1, \quad U(1)=0, \quad U(-y) \equiv U(y) .
$$

The disturbance imposed upon this flow is to be taken as an elementary wave of length $2 \pi / \alpha$, in the direction of flow.

The problem as stated is known ${ }^{2}$ to admit of formulation as the differential boundary problem

\footnotetext{
1 Presented to the Society, September 7, 1939.

2 C. L. Pekeris, On the stability problem in hydrodynamics, I, Proceedings of the Cambridge Philosophical Society, vol. 32 (1936), p. 55, and II, Journal of the Aeronautical Sciences, vol. 5 (1938), p. 236.
} 


$$
\begin{aligned}
& f^{\mathrm{iv}}(y)-2 \alpha^{2} f^{\prime \prime}(y)+\alpha^{4} f(y) \\
& +i \alpha R\left[\{U(y)-C\}\left\{f^{\prime \prime}(y)-\alpha^{2} f(y)\right\}-U^{\prime \prime}(y) f(y)\right]=0, \\
& f(1)=f(-1)=f^{\prime}(1)=f^{\prime}(-1)=0 .
\end{aligned}
$$

Of the constants involved in this, $\alpha$ has already been defined and $R$ is the Reynold's number. $C$ is a complex parameter for characteristic values of which the boundary problem admits of solution. In terms of such a solution the stream function of the associated flow is given by the formula $f(y) e^{i \alpha(C t-x)}$. This flow is accordingly stable if and only if the imaginary component of the characteristic value $C$ is positive.

In the special case of a parabolic flow, that is, $U(y)=1-y^{2}$, the problem has recently been considered by Pekeris. ${ }^{3}$ His deductions apply in the main to cases in which the Reynold's number is suitably small, it being shown in particular that stability always obtains if this number does not exceed the smaller of the numbers $2 \alpha$ and $\alpha^{3}$. The developments of the smaller characteristic values in series of powers of $\alpha R$ are analyzed, and it is also shown that a symmetrical flow is stable in the limiting case as $\alpha \rightarrow 0$. The case of more general velocity profiles $U(y)$ has been considered, among others by Tollmien. ${ }^{4}$ His work has special reference to cases in which the Reynold's number is large enough to make $1 / \alpha R$ generally negligible, and is focused upon characteristic values with imaginary components that are small.

The present discussion differs widely from those cited. It is of an asymptotic nature and is concerned with the characteristic values for which the product $\alpha R|C|$ is large. The results may be regarded, therefore, as applying to flows associated with large characteristic values if $\alpha R$ is small or moderate, and to characteristic values which are large, moderate, or even small if $\alpha R$ is large. The values of $\alpha$ and $R$, which are real and positive, are regarded as either fixed or variable. In the latter case the ranges of $\alpha$, and of $1 / \alpha R$, are to be taken as bounded. It will be shown that any flow for which these hypotheses are fulfilled, and for which the imaginary component of $C$ is not very small, is stable.

In the complex plane of the parameter $C$, let the region consisting of the infinite strip

$$
0<\Re(C)<1, \quad \Im(C)>\because 0,
$$

plus an arbitrarily small but fixed neighborhood of the segment of the

${ }^{3}$ Loc. cit. Reference is made to these papers for further citations of the literature.

${ }^{4} \mathrm{~W}$. Tollmien, Ein allgemeines Kriterium der Instabilität laminarer Geschwindigkeitsverteilungen, Nachrichten von der Gesellschaft der Wissenschaften zu Göttingen, 1935, p. 79. 
axis of reals from 0 to 1 , be shaded, as is shown in Fig. 1 . It will be assumed throughout the pending discussion that $C$ lies in the unshaded region, and it will be shown that under this assumption no

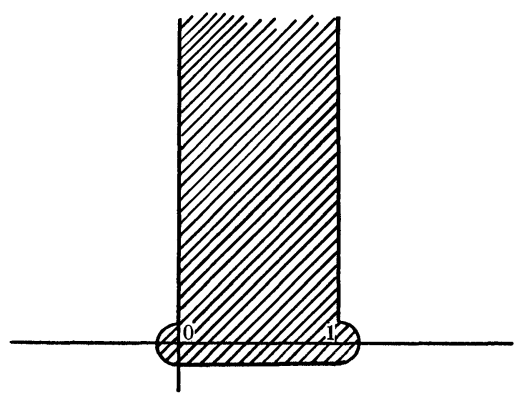

FIG. 1

solution of the boundary problem (1) exists. The inference will then be clear that all characteristic values lie in the shaded region.

In terms of the abbreviations

$$
\begin{aligned}
\rho & =|\alpha R C|^{1 / 2} \exp \{i(\pi / 4+(1 / 2) \arg C)\}, \\
\phi(y) & =[1-U(y) / C]^{1 / 2}, \quad \phi(1)=1, \\
\kappa(y) & =U^{\prime \prime}(y) / C,
\end{aligned}
$$

the differential equation (1) takes the form

(4) $f^{\text {iv }}-\left\{\rho^{2} \phi^{2}(y)+2 \alpha^{2}\right\} f^{\prime \prime}+\left\{\rho^{2}\left[\alpha^{2} \phi^{2}(y)-\kappa(y)\right]+\alpha^{4}\right\} f=0$.

The range of the variable is $-1 \leqq y \leqq 1$. Since $U(y)$ is real and takes only values from 0 to 1 , whereas $C$ is located in the unshaded portion of the complex plane, the functions $\kappa(y), \phi(y), 1 / \phi(y)$ are bounded.

If an expression

$$
\exp \left\{\rho \int_{0}^{y} \phi(y) d y\right\} P(y, \rho),
$$

in which $P(y, \rho)$ is an undetermined power series in $1 / \rho$, that is,

$$
P(y, \rho) \equiv p_{0}(y)+p_{1}(y) / \rho+p_{2}(y) / \rho^{2}+\cdots,
$$

is substituted for $f$, the left-hand member of (4) takes the form of an exponential multiplied by a power series in $1 / \rho$ with coefficients that are functions of $y$. By equating these coefficients to zero a sequence of differential equations is obtained which involve the functions $p_{j}(y), j=0,1,2, \cdots$, and from which these functions are successively determinable by integrations. The constants which enter by 
virtue of these integrations may be chosen, and are to be chosen, so that the functions $p_{j}(y)$ with even subscripts are even functions of $y$, and those with odd subscripts are odd functions of $y$, and so that $p_{0}(1)=1$. Those constants which remain undetermined by these stipulations may be chosen at pleasure, since their choice has no effect upon the deductions which are to be made. With the determinations described, the expression (5) is a formal solution of the differential equation (4), and formally also $P(-y, \rho) \equiv P(y,-\rho)$. Inasmuch as the equation (4) involves $\rho$ only in the form $\rho^{2}$, it is clear also that with this determination of $P(y, \rho)$ the expression

$$
\exp \left\{-\rho \int_{0}^{y} \phi(y) d y\right\} P(y,-\rho)
$$

is likewise a formal solution of (4).

If, now, in a similar manner, an undetermined series

$$
S(y, \rho) \equiv s_{0}(y)+s_{1}(y) / \rho^{2}+s_{2}(y) / \rho^{4}+\cdots
$$

is substituted for $f$, the left-hand member of (4) takes the form of a power series in $1 / \rho^{2}$. Equating its coefficients to zero leads to the sequence of relations

$$
\begin{aligned}
& \phi^{2} s_{0}^{\prime \prime}-\left(\alpha^{2} \phi^{2}-\kappa\right) s_{0}=0 \\
& \phi^{2} s_{j}^{\prime \prime}-\left(\alpha^{2} \phi^{2}-\kappa\right) s_{j}=s_{j-1}^{\text {iv }}-2 \alpha^{2} s_{j-1}^{\prime \prime}+\alpha^{4} s_{j-1}, \quad j=1,2,3, \cdots .
\end{aligned}
$$

These equations may be solved by functions $s_{j}(y)$ determined successively as odd functions of $y$, say as functions $s_{j, 0}(y), j=0,1,2, \cdots$. They may, however, also be solved by functions determined successively as even functions of $y$, say as functions $s_{j, e}(y), j=0,1,2, \cdots$. If this is done, the resulting series

$$
\begin{aligned}
& S_{0}(y, \rho) \equiv s_{0,0}(y)+s_{1,0}(y) / \rho^{2}+s_{2,0}(y) / \rho^{4}+\cdots, \\
& S_{e}(y, \rho) \equiv s_{0, e}(y)+s_{1, e}(y) / \rho^{2}+s_{2, e}(y) / \rho^{4}+\cdots
\end{aligned}
$$

are respectively odd and even, and are formal solutions of the equation (4).

The function $U(y)$ takes on only real values from 0 to 1 . For $C$ located anywhere in the unshaded part of the plane in Fig. 1, it accordingly follows that

$$
-3 \pi / 2 \leqq \arg [C-U(y)] \leqq \pi / 2,
$$

and hence, by (3), that

$$
-\pi / 2 \leqq \arg \rho \phi(y) \leqq \pi / 2 .
$$


This, however, is a familiar sufficient condition ${ }^{5}$ that the formal expressions (5), (7), and (9), which are in general divergent, have the property of each representing asymptotically some solution of the differential equation (4). There exist, therefore, solutions $f_{j}(y, \rho)$, $j=1,2,3,4$, of this equation, for which the representations

$$
\begin{aligned}
& f_{1}(y, \rho) \sim \exp \left\{\rho \int_{0}^{y} \phi(y) d y\right\} P(y, \rho) \\
& -\exp \left\{-\rho \int_{0}^{y} \phi(y) d y\right\} P(y,-\rho), \\
& f_{2}(y, \rho) \sim \exp \left\{\rho \int_{0}^{y} \phi(y) d y\right\} P(y, \rho) \\
& \quad+\exp \left\{-\rho \int_{0}^{y} \phi(y) d y\right\} P(y,-\rho), \\
& f_{3}(y, \rho) \sim S_{\theta}(y, \rho)
\end{aligned}
$$

are valid. Of these solutions $f_{1}$ and $f_{3}$ are odd functions of $y$, and $f_{2}$ and $f_{4}$ are even.

Any solution of the boundary problem (1) is either even or odd; that is, the flow associated with it is either symmetrical or anti-symmetrical. Let it be assumed, to begin with, that there exists such an odd solution. Since it satisfies the differential equation (1), it may be represented in terms of the solutions (11) in the manner $c_{1} f_{1}(y)+c_{3} f_{3}(y)$, with coefficients $c_{1}, c_{3}$ which are independent of $y$, and, since it satisfies the boundary conditions (1), this and its derivative vanish at $y=1$. Inasmuch as $c_{1}$ and $c_{3}$ are not both zero if the solution is nontrivial, it follows that the determinant

$$
\left|\begin{array}{ll}
f_{1}(1, \rho) & f_{3}(1, \rho) \\
f_{1}^{\prime}(1, \rho) & f_{3}^{\prime}(1, \rho)
\end{array}\right|
$$

must be zero, a relation which in virtue of the formulas (11), and the evaluations $\phi(1)=p_{0}(1)=1$, takes the form

$$
\begin{aligned}
\exp \left\{2 \rho \int_{0}^{1} \phi(y) d y\right\} & \\
& \cdot\left\{\frac{\left[\rho P(1, \rho)+P^{\prime}(1, \rho)\right] S_{0}(1, \rho)-P(1, \rho) S_{0}^{\prime}(1, \rho)}{\left[-\rho P(1, \rho)+P^{\prime}(1, \rho)\right] S_{0}(1, \rho)-P(1, \rho) S_{0}^{\prime}(1, \rho)}\right\} \sim 1 .
\end{aligned}
$$

${ }^{5} \mathrm{H}$. L. Turrittin, Asymptotic solutions of certain ordinary differential equations ..., American Journal of Mathematics, vol. 58 (1936), p. 364, 
This, it is found, is reducible to the form

$$
\rho \int_{0}^{1} \phi(y) d y+A_{1} / \rho+A_{2} / \rho^{2}+\cdots \sim B_{n} i,
$$

in which $n$ is an appropriate integer, and in which

$$
A_{1}=p_{0}^{\prime}(1)-s_{0,0}^{\prime}(1) / s_{0,0}(1), \quad B_{n}=(n+1 / 2) \pi,
$$

if for the case in hand $s_{0,0}(1) \neq 0$, while

$$
A_{1}=-s_{1,0}(1) / s_{0,0}^{\prime}(1), \quad B_{n}=n \pi,
$$

if $s_{0,0}(1)=0$ (and hence $s_{0,0}^{\prime}(1) \neq 0$ ). Since $|\rho|$ is assumed to be large, the numerical values of $n$ and $B_{n}$ are evidently also large.

Let $M$ be chosen as any sufficiently large real positive constant. The values of $C$ may be considered then in the categories $|C| \geqq M$, and $|C|<M$, and it is convenient to do this. If $|C| \geqq M$, the expansions

$$
\phi(y)=1-U(y) / 2 C+\cdots, \quad A_{1}=a_{0}+a_{1} / C+\cdots
$$

may be used, and simple calculations show that the constant $a_{0}$ is real. The relation obtained by squaring the members of (12) is then found to be

$$
C-\int_{0}^{1} U(y) d y-2 i a_{0} / \alpha R+O(1 / C)+O(1 / \rho)=B_{n}^{2} i / \alpha R .
$$

To satisfy this the value $C$ must evidently have a positive imaginary component, and, if $M$ has been chosen suitably large, a real component which differs so little from the value $\int_{0}^{1} U(y) d y$ that it lies between the values 0 and 1 . The relation is, therefore, impossible for the values of $C$ here in question since they lie in the unshaded part of the plane of Fig. 1.

If $|C|<M$ on the other hand, let $y_{1}$ and $y_{2}$ be chosen in any way such that $0<y_{1}<y_{2}<1$ and $U\left(y_{1}\right)<1, U\left(y_{2}\right)>0$, and let $\epsilon$ designate the smaller of the values $1-U\left(y_{1}\right), U\left(y_{2}\right)$. Then since $C$ lies in the unshaded part of the plane, it follows that when $y_{1} \leqq y \leqq y_{2}$ then

$$
-\pi-\tan ^{-1} M / \epsilon \leqq \arg [C-U(y)]<\tan ^{-1} M / \epsilon,
$$

namely that

$$
|\arg \rho \phi(y)| \leqq \pi / 2-(1 / 2) \cot ^{-1} M / \epsilon .
$$

Thus, on the interval $\left(y_{1}, y_{2}\right)$

$$
\Re(\rho \phi(y)) \geqq|\rho \phi(y)| \sin \left((1 / 2) \cot ^{-1} M / \epsilon\right),
$$


and since by (10) this real part is not negative even outside the interval concerned, it follows that

$$
\Re\left\{\rho \int_{0}^{1} \phi(y) d y\right\} \geqq|\rho| \sin \left((1 / 2) \cot ^{-1} M / \epsilon\right) \int_{y_{1}}^{y_{2}}|\phi(y)| d y .
$$

The leading term in the left-hand member of (12) accordingly has a real part which in magnitude is of the order of $|\rho|$. Inasmuch as the right-hand member is pure imaginary, the relation is clearly impossible.

It has thus been shown that all characteristic values for which the flow of the fluid is anti-symmetrical must lie in the shaded part of the plane of Fig. 1. In like manner the same result may be obtained for the characteristic values associated with symmetrical flows. Except for possible flows in the immediate border region between stability and instability, that is, for which the imaginary component of $C$ is very small, it must be inferred, therefore, that any flow for which $\alpha R|C|$ is large is stable.

UNIVERSITY OF WISCONSIN 